keine weitgehenden Schlüsse ziehen; doch wird man auch nach weiteren Versuchen keine Normen aufstellen können. Auffallend ist in einzelnen Fällen der verhältnismäßoig hohe Säuregrad der Milch, eine Tatsache, die sich mit den Angaben einzelner Produzenten deckt, daß die Milch während der Brunst der Tiere leicht gerinne. Bei keinem der Versuche war, und das ist wichtig, eine auffallende Veränderung der Milch. im Gehalte an fettfreier Trockensubstanz zu beobachten, eine Tatsache, die im Hinblick auf das eingangs erwähnte Schutzvorbringen immerhin einigermaßen beruhigend zu wirken geeignet ist. Allerdings ist zu berücksichtigen, daß es sich wenigstens bei den Kühen No. III-VIII um Tiere handelte, die nur trocken gefüttert wurden. Leider hatten und haben wir hier keine Gelegenheit, diese Versuche häufiger und besonders bei Tieren, die nur Grünfutter erhalten, zu wiederholen. Wir können daher nur wünschen, daß diese Ausführungen vielleicht dem einen oder andern Fachgenossen, dem solche Stallungen zur Verfügung stehen, Auregung geben, diese Verhältnisse dort weiter zu prüfen. Denn für die sichere Beurteilung der Einzelmilch erscheint uns die Beibringung weiteren Materiales nach dieser Richtung außerordentlich wertvoll. Überhaupt wäre zu wünschen, daf besonders auch in allen denjenigen Fällen, wo einwandfreie Stallprobenmilch, wie ich aus unserer Erfahrung oben einige mitgeteilt babe, auffallend niedrige Werte für das spezifische Gewicht des Serums und die fettfreie Trockensubstanz zeigen, diese Verhältnisse bei den betreffenden Kühen solange weiter verfolgt würden, bis man Klarheit darüber hat, ob bier nur plötzlich aufgetretene Schwankungen vorliegen, oder ob die betreffenden Tiere ständig eine solche abnorme Milch liefern. Denn wäre ersteres der Fall, so würde bei geringeren Schwankungen zwisehen Stallprobenmilch und verdächtiger Probe die erstere bedeutend an Beweiskraft einbüBen.

\title{
Über die Kontrolle der natürlichen Mineralwässer auf Echtheit.
}

\author{
Von \\ Otto Mezger und Karl Grieb.
}

Mitteilung aus dem Chemischen Laboratorium der Stadt Stattgart.

(Direktor: Dr. Bujard.)

Vor einiger Zeit gab ein Spezjalfall unserem Institute Veranlassung, sich eingehender mit der Untersuchung einer grösseren Anzahl von hier im Handel befindlichen Mineralwässern auf Echtheit zu befassen. Anläßlich der Besichtigung einer Sollawasserfabrik fiel uns nämlich bei dem in dem Betrieb aufgestapelten Vorrat an Teinacher- und Uberkinger-Wasser auf, daß einzelne Flaschen keine, oder nur mangeihafte Verschlubstreifen trugen. Bei näherer Untersuchung dieser Verschlußstreifen zeigte es sich, daß diese aus Bruchstücken bereits benutzter Streifen mit. Hilfe von Stärkekleister zusammengeklebt waren. Eine große Anzahl, das wollen wir vorsusschicken, der in dem betr. Betrieb angetroffenen Teinacher-Flaschen enthielten den wenig bekannten Löwensprudel, den der Betriebsinhaber von seinem Vater, dem Besitzer dieser Quelle, billig bezog und in die Teinacher - Flaschen umfüllte. Die ÜberkingerFlaschen enthielten dagegen meistens gewöhnliches Sodawasser. Es sei noch bemerkt, daß an Hand der Bücher auch noch eine große Anzahl von Abnehmern, meist Gast- 
wirte, festgestellt werden konnte, denen der Betriebsinhaber in größerem Maßstab ebenfalls diese gefälschten Wässer für echte geliefert hatte. Der Fall ist bereits abgeurteilt ${ }^{1}$ ). Vielleicht gibt der Fall dem einen oder andern Fachgenossen Veranlassung, sein Augenmerk auch auf den Handel mit natürlichen Mineralwässern zu lenken. Für die schnelle Orientierung über die Echtheit einiger der hauptsächlichsten bei uns gehandelten natürlichen Mineralwässer konnten wir anläßlich dieser Massenuntersuchung wertvolle Gesichtspunkte gewinnen. Zunächst mag die tabellarische Übersicht über die in den letzten Jahren überhaupt und die anläblich dieses Spezialfalles in unserem Institute ausgeführten Untersuchungen von Mineralwässern folgen.

J. Als Teinacher Sprudel bezeichnete Wässer.

1. Echter Teinacher Sprudel. Verschlubstreifen unverletzt.

\begin{tabular}{|c|c|c|c|c|c|c|c|c|c|c|c|}
\hline \multirow[b]{2}{*}{ No. } & \multirow{2}{*}{$\begin{array}{c}\text { Zeit } \\
\text { der Ent- } \\
\text { nahme }\end{array}$} & \multicolumn{3}{|c|}{ In 1 Liter Wasser } & \multirow{2}{*}{$\begin{array}{c}\text { Voruber- } \\
\text { gehende } \\
\text { Härte } \\
\text { (Dentsche } \\
\text { Grade) }\end{array}$} & \multirow[b]{2}{*}{ No. } & \multirow{2}{*}{$\begin{array}{c}\text { Zeit } \\
\text { der Ent- } \\
\text { nahme }\end{array}$} & \multicolumn{3}{|c|}{ In 1 Liter Wasser } & \multirow{2}{*}{$\begin{array}{c}\text { Vorüber- } \\
\text { gehende } \\
\text { Härte } \\
\text { (Deutsehe } \\
\text { Grade) }\end{array}$} \\
\hline & & $\begin{array}{c}\text { Trocken- } \\
\text { rückstand } \\
\mathrm{mg}\end{array}$ & $\begin{array}{c}\text { Glüh- } \\
\text { ruiekstand } \\
\text { mg }\end{array}$ & $\begin{array}{c}\text { Chlor } \\
\mathrm{mmg}\end{array}$ & & & & $\begin{array}{c}\text { Trocken- } \\
\text { ruckstand } \\
\text { mg }\end{array}$ & $\begin{array}{c}\text { Glüh- } \\
\text { ruickstand } \\
\text { mg }\end{array}$ & $\begin{array}{l}\text { Chlor } \\
\text { mg }\end{array}$ & \\
\hline 1 & 1903 & 1482 & 1402 & 35,5 & 67,2 & 12 & 1907 & - & - & 35,0 & 64,4 \\
\hline 2 & 1904 & 1332 & 1206 & 39,0 & & 18 & 190 & - & - & 36,0 & 66,1 \\
\hline 3 & 1904 & - & - & 35,5 & 63 & 14 & 1908 & - & - & 35,0 & 64,4 \\
\hline 4 & 190 & - & - & 35,5 & & 15 & 1908 & - & - & 36,0 & 66,1 \\
\hline 5 & 1904 & - & - & 35,5 & & 16 & 1907 & 1384 & 1244 & 39,0 & 65,0 \\
\hline 6 & 1905 & - & - & 39,0 & 63 & 17 & 1907 & 1378 & 1278 & 37,7 & 65,0 \\
\hline 7 & 1905 & - & - & 35,5 & 68,8 & 18 & 1907 & 1375 & 1270 & 39 & 65,0 \\
\hline 8 & 190 & - & - & 39,0 & 64 & 19 & 1907 & $1836^{\circ}$ & 1224 & 37,7 & 61,6 \\
\hline 9 & 1907 & - & - & 41,0 & 66,1 & 20 & 1907 & - & - & 39,0 & 66,1 \\
\hline 10 & 1907 & - & - & 41,0 & 67,2 & 21 & 1907 & - & - & 39,0 & 65,0 \\
\hline 11 & 1907 & - & - & 35,5 & 65,0 & & & & & & \\
\hline
\end{tabular}

2. In Teinacher Flaschen umgefüllter Löwen-Sprudel. Verschlußstreifen zusammengeklebt aus Bruchstücken von schon benutzten Teinacher Streifen.

\begin{tabular}{|c|c|c|c|c|c|c|c|c|c|c|}
\hline 1907 & - & - & 14,2 & 39,2 & 39 & 1907 & - & - & 17,7 & 39,8 \\
\hline 23 & - & - & 14,2 & 38,6 & 40 & $\pi$ & - & - & 14,2 & 39,2 \\
\hline 24 & - & - & 14,2 & 38,1 & 41 & n & 一 & - & 14,2 & 39,2 \\
\hline 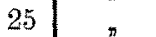 & $\ldots$ & - & 14,2 & 38,6 & 42 & 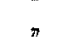 & - & - & 14,2 & 39,2 \\
\hline , & - & - & 10,6 & 37,0 & 43 & $n$ & - & - & 14,2 & 39,2 \\
\hline , & - & - & 14,2 & 38,1 & 44 & $\pi$ & - & - & 14,2 & 39,2 \\
\hline 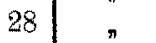 & - & - & 14,2 & 39,2 & 45 & $n$ & - & - & 14,2 & 39,8 \\
\hline 29 & - & - & 14,2 & 38,6 & 46 & n & - & - & 14,2 & 39,2 \\
\hline 80 & - & - & 14,2 & 39,2 & 47 & $\pi$ & - & - & 10,6 & 39,8 \\
\hline 31 & - & - & 16,0 & 37,0 & 48 & , & - & - & 14,2 & 39,8 \\
\hline 32 & - & - & 16,0 & 38,4 & 49 & , & - & - & 10,6 & 38,6 \\
\hline 33 & - & - & 14,2 & 39,8 & 50 & , & - & - & 14,2 & 39,2 \\
\hline 34 & - & - & 14,2 & 39,2 & 51 & , & 869 & 794 & 14,2 & 38,1 \\
\hline 35 & - & - & 14,2 & 38,1 & 52 & 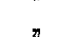 & - & - & 14,2 & 38,1 \\
\hline 36 & - & - & 14,2 & 39,8 & 53 & $"$ & - & - & 10,6 & 37,0 \\
\hline 37 & 927 & 860 & 14,2 & 39,8 & 54 & $"$ & - & - & 10,6 & 38,1 \\
\hline n & - & - & 14,2 & 39,2 & & & & & & \\
\hline
\end{tabular}

1) Vergl, diese Zeitschrift 1908, 16, 332. 
II. Echter Löwen-Sprudel aus Obernau.

Ohne Verschlufostreifen; Proben aus Bahnsendungen entnommen.

\begin{tabular}{|c|c|c|c|c|c|c|c|c|c|c|c|}
\hline \multirow[b]{2}{*}{ No. } & \multirow{2}{*}{$\begin{array}{c}\text { Zeit } \\
\text { der Ent- } \\
\text { nahme }\end{array}$} & \multicolumn{3}{|c|}{ In 1 Liter Wasser } & \multirow{2}{*}{$\begin{array}{l}\text { Vorüber- } \\
\text { gehende } \\
\text { Härte } \\
\text { (Dentsehe } \\
\text { Grade) }\end{array}$} & \multirow[b]{2}{*}{ No. } & \multirow{2}{*}{$\begin{array}{c}\text { Zeit } \\
\text { der Ent- } \\
\text { nahme }\end{array}$} & \multicolumn{3}{|c|}{ In 1 Liter Wasser } & \multirow{2}{*}{$\begin{array}{l}\text { Vorüber- } \\
\text { geliende } \\
\text { Härte } \\
\text { (Dentsehe } \\
\text { Grade) }\end{array}$} \\
\hline & & $\begin{array}{l}\text { Trocken- } \\
\text { rǘkstand } \\
\text { mg }\end{array}$ & $\left|\begin{array}{c}\text { Glühl- } \\
\text { rïekstand } \\
\mathrm{mg}\end{array}\right|$ & $\begin{array}{c}\text { Cblor } \\
\text { mg }\end{array}$ & & & & $\left|\begin{array}{c}\text { Trocken- } \\
\text { rückstand } \\
\text { mig }\end{array}\right|$ & $\begin{array}{c}\text { Glüh- } \\
\text { rüekstand } \\
\text { mg }\end{array}$ & $\begin{array}{c}\text { Chlor } \\
\text { mg }\end{array}$ & \\
\hline 55 & 1907 & 934 & 865 & 14,2 & 42,0 & 60 & 1907 & 869 & 794 & 14,2 & 38,1 \\
\hline 56 & 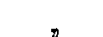 & - & - & 14,2 & 42,6 & 61 & \# & - & - & 14,2 & 42,0 \\
\hline 57 & n & - & - & 14,2 & 43,7 & 62 & n & - & - & 14,2 & 39,0 \\
\hline 58 & $n$ & - & - & 14,2 & 43,7 & 63 & $\pi$ & - & - & 16,0 & 38,9 \\
\hline 59 & $\pi$ & 979 & 900 & 14,2 & 43,7 & 64 & , & - & - & 14,2 & 39,8 \\
\hline
\end{tabular}

III. Als Überkinger Sprudel bezw. Überkinger Wasser bezeichnete Wässer.

1. Echter Überkinger Sprudel. Verschlufstreifen unverletzt.

\begin{tabular}{c|c|c|c|c|c|c|c|c|c|c|c}
65 & 1907 & 1506 & 1446 & 147 & 67,8 & 70 & 1907 & 1532 & 1485 & 149 & 70,9 \\
66 & $"$ & - & - & 114 & 63,8 & 71 & $n$ & - & - & 163 & 63,3 \\
67 & $"$ & - & - & 121 & 63,8 & 72 & $*$ & - & - & 185 & 66,1 \\
68 & $*$ & - & - & 129 & 64,4 & 73 & $*$ & - & - & 199 & 66,1 \\
69 & $"$ & 1460 & 1411 & 170 & 66,1 & & & & & &
\end{tabular}

2. Gew ohnliches Überkinger Wasser. Verschlußsstreifen unverletzt.

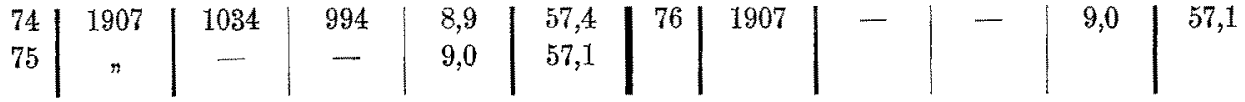

3. Als Überkinger Sprudel bezeichnetes Sodawasser. Ohne Verschlufstreifen.

\begin{tabular}{|c|c|c|c|c|c|c|c|c|c|c|}
\hline \begin{tabular}{l|l}
77 & 1907
\end{tabular} & 22371 & 1751 & 763 & 18,0 & 86 & 1907 & 1756 & 1691 & 760 & $\begin{array}{l}17,9 \\
\end{array}$ \\
\hline 78 & 1708 & 1631 & 781 & 19,6 & 87 & \# & - & - & 768 & 18,2 \\
\hline 79 & 1760 & 1651 & 781 & 19,3 & 88 & , & - & 一 & 852 & 18,5 \\
\hline 80 & - & - & 781 & 19,3 & 89 & , & - & - & 738 & 17,9 \\
\hline 81 & - & - & 781 & 19,6 & 90 & " & 1707 & 1643 & 753 & 17,6 \\
\hline 82 & - & - & 769 & 19,6 & 91 & n & - & - & 738 & 17,4 \\
\hline 83 & - & - & 767 & 19,6 & 92 & n & - & - & 724 & 17,0 \\
\hline 84 & - & - & 753 & 18,5 & 93 & $n$ & - & - & 795 & 19,0 \\
\hline 85 & - & - & 763 & 19,0 & & & & & & \\
\hline
\end{tabular}

IV. Echtes Göppinger Wasser. Verschlufstreifen unverletzt.

\begin{tabular}{c|c|c|c|c|c|c|c|c|c|c|c|c}
94 & 1907 & 1035 & 983 & 10,6 & 54,9 & 96 & 1907 & 1038 & 964 & 10,6 & 54,3 \\
95 & " & 1034 & 994 & 10,6 & 54,9 & & & & & &
\end{tabular}

V. Leitungs w as er der Sodawasserfabrik.

$97|1907|-|-| 14,2|17,6|+|| \mid$

Zur annähernden Bestimmung der vorübergehenden Härte wurden jeweils $200 \mathrm{ccm}$ Wasser nach P feifer-Lunge ohne Erwärmung mit $1 / 5$ N.-Salzsäure unter Verwendung von Methylorange als Indikator auf die Übergangsfarbe titriert; die

1) Das Wasser enthält Zucker. 
verbrauchten ccm, mit 2,8 multipliziert, geben bekanntlich einen ungefähren Wert für die voräbergehende Härte in deutschen Härtegraden. Der Chlorgehalt wurde titrimetrisch nach $\mathrm{Mohr}$ ermittelt.

Was die als echt in den Tabellen bezeichneten Wässer anbelangt, so wurden solche teils direkt von den Quellen bezogen, teils aus Bahnsendungen und teils aus verschiedenen als durchaus zuverlässig bekannten Geschäften entnommen. Bezüglich der Beurteilung der einzelnen Wassersorten sei folgendes ausgeführt:

\section{Teinacher Wasser.}

Die Alkalität der als echt erkannten Wässer (No. 1-21), ausgedrückt als vorübergehende Härte, bewegte sich etwa zwischen 62 und 67 Graden, der Chlorgehalt schwankte etwa zwischen 35 und $41 \mathrm{mg}$ im Liter. Beide Werte sind aber ziemlich konstant. Als gefälscht wurden dagegen erkannt die Proben No. 22-54. Bei ihnen schwankte die vorübergehende. Härte etwa zwischen 37 und 40 Graden, der Chlorgehalt etwa zwischen 11 und $16 \mathrm{mg}$ : Das in den Teinacher-Flaschen mit aus Bruchstücken zusammengeklebten Verschlußstreifen enthaltene Wasser war somit, wie ein Vergleich mit den Proben No. 55-64 zeigt, offenbar Löwen-Sprudel.

\section{Löwen-Sprudel.}

Als echt wurden erkannt die Proben No. 55-64; bei ibnen schwankte die vorübergehende Härte etwa zwischen 38 und $44^{\circ}$ und der ChIorgehalt zwischen 14 und $16 \mathrm{mg}$ im Liter.

\section{Uberkinger Wasser.}

Als echt wurden erkannt die Proben No. 65-76. Die vorübergehende Härte schwankte bei ihnen etwa zwischen 57 und $71^{\circ}$. Was den Chlorgehalt anbelangt, so schwankt derselbe bei dem Überkinger Sprudel etwa zwischen 114 und $200 \mathrm{mg}$ im Liter; bei den drei untersuchten Proben des gewöhnlichen Überkinger Wassers (No. 74-76) beträgt ex durchweg etwa $9 \mathrm{mg}$. Demnach liegt der Schluß nahe, daß bei der Herstellung des'Sprudels Chloride, und zwar jedenfalls in der Hauptsache Kochsalz zugesetzt werden.

Die Proben No. 77-93 stellten kein echtes Überkinger Wasser, sondern lediglich ein aus Leitungswasser unter Zusatz von Chloriden hergestelltes Sodawasser vor. Die vorübergehende Härte schwankte bei diesen Proben, etwa zwischen 18 und $20^{\circ}$, der Chlorgehalt etwa zwischen 740 und $850 \mathrm{mg}$ im Liter. Das Leitungswasser in dem betreffenden Betriebe (No. 97) zeigte $17,6^{\circ}$ vorübergehende Härte; es zeigt also diese Probe gegenüber den Proben No. 77-93 in der vorübergehenden Härte keine große Abweichung; diese ist offenbar auf die Einwirkung der bei der Bereitung des Sodawassers verwendeten Salze zurückzuführen.

\section{Göppinger Wasser.}

Es gelangten nur echte Wässer zur Untersuchung; ihre vorübergehende Härte schwankte zwischen 54,3 und 54,90, ihr Chlorgehalt betrug durchweg 10,6 $\mathrm{mg}$ im Liter

Aus den obigen Ausführungen geht hervor, daß die Alkalität der verschiedenen unte suchten natürlichen Mineralwässer eine Konstante darstellt, die sich rasch ermitteln 
läßt und die gegenüber dem Chlorgehalt einen viel zuverlässigeren Wert hinsichtlich dex Unterscheidung bezw. Prüfung auf Echtheit abgibt. Wie aus den beim Überkinger Wasser erwähnten bedeutenden Schwankungen im Chlorgehalt hervorgeht, wird der Überkinger Sprudel offenbar unter Zusatz von Chloriden (jedenfalls in der Hauptsache Kochsalz) hergestellt, sodal die titrimetrische Bestimmung des Chlorgehalts zur raschen Orientierung bei gewissen Sprudelwässern jedenfalls nicht den Wert besitzt, wie die Bestimmung der Alkalität.

Daß die Alkalität durch den Zusatz von Salzen je nach ihrer Art und unter Umständen nach ihrem Reinheitsgrad in erheblichem Maße beeinflubt wird, liegt auf der Hand, es geht dies auch aus der Tabelle insofern hervor, als alle diejenigen Wässer, bei denen große Schwankungen in Chlorgehalt zu beobachten sind, auch in der Alkalität wesentlich mehr schwanken, als die Wässer, bei denen der Chlorgellalt innerhalb engerer Grenzen liegt. Eine wirkliche Konstanz in der Alkalität wird man daher nur bei denjenigen wirklichen Mineralwässern haben, die ohne Zusätze in den Handel kommen und daher auch in in Wirklichkeit „rein natürliche Mineralwässer"rs sind.

\section{Über die Volumenbestimmung von Gebäckstücken.}

Von

\section{P. Neumann und P. Salecker.}

Mitteilung aus der Versuchsanstalt für Getreideverarbeitung in Berlin.

Für die Bewertung des Mehles in bäckereitechnischer Hinsicht ist die Volumenmessung der aus dem Mebl gewonnenen Gebäckstücke fast die einzige Bestimmung, die in Zahlen ausdrückbare Ergebnisse liefert. Es ist daher allgemein üblich, diese Volumenmessung vorzunehmen, zumal es auch dem Praktiker gewöhnlich in erster Linie darauf ankommt, zu erfahren, ob das zu bewertende Mehl ein ins Auge fallendes Gebäck von möglichst großem Umfang liefert. Es kann nicht fraglich sein, daß dieser Wert eine gewisse Berechtigung hat, da mit einer großen Volumenausdehnung des Gebäckes eine bessere Lockerung der Krume und somit eine größere Bekömmlichkeit parallel geht. Andererseits muß aber nachdrücklich eine ganz einseitige auf die Volumgröße der Gebäcke gerichtete Beurteilung des Mehles, wie sie in letzter Zeit Platz gegriffen hat, als verfehlt bekämpft werden. Vor allem ist es notwendig, jedes Gebäckstück nach der Volumenbestimmung auf seine Porenverteilung in der Krume zu prüfen, da die häufig im Innern der Gebäcke sich findenden „Gärblasen“ einen scheinbaren hohen Wert für dié durch gute Porosität bedingte Vollimengröße ergeben, der in der Tat für die Bewertung des Mehles belanglos ist oder gar irreführende Bedeutung erhält. Es ist bei dieser Prüfung der Porenverteilung gewöhnlich nicht notwendig, die Porengröße oder das Porenvolumen der Krume zu ermitteln, vielmehr genügt die augenscheinliche Feststellung gleichmäßiger Verteilung. Nur solche Gebäckstücke dürfen zum Vergleich herangezogen werden, deren Krume nicht mit g röß e r en Lufträumen, „Gärblasen“, durchsetzt ist.

Die Volumenmessung der Gebäcke wird nun in der bekannten Weise durch 